\title{
Dexmedetomidine protects against sepsis-associated encephalopathy through Hsp90/AKT signaling
}

\author{
LIJUN YIN, XUEJUN CHEN, HONGBO JI and SHUNLI GAO
}

\author{
Department of Anesthesiology, Tianjin Baodi Hospital, \\ Baodi Clinical College of Tianjin Medical University, Tianjin 301800, P.R. China
}

Received April 9, 2019; Accepted September 4, 2019

DOI: $10.3892 / \mathrm{mmr} .2019 .10718$

\begin{abstract}
Sepsis-associated encephalopathy (SAE) is characterized by neuronal apoptosis and changes in mental status. Accumulating evidence has. indicated that dexmedetomidine is capable of protecting the brain against external stimuli and improving cognitive dysfunctions. The aim of the present study was to investigate the possible neuroprotective effects of dexmedetomidine on SAE and the role of heat-shock protein (Hsp)90/AKT signaling in an experimental model of sepsis. The SAE model was established by cecal ligation and perforation (CLP) in vivo and lipopolysaccharide (LPS) treated hippocampal neuronal cultures in vitro. It was found that dexmedetomidine inhibited caspase-3, but increased the expression level of Bcl-2 in CLP rats. CLP rats also exhibited a decreased level of phosphorylated AKT Thr 308 and Hsp90, and their expression could be reversed by treatment with dexmedetomidine. Additionally, application of dexmedetomidine increased cell survival and decreased neuronal apoptosis in vitro. Furthermore, the neuroprotective effects of dexmedetomidine could be reversed by 17-AAG (a Hsp90 inhibitor), or wortmannin (a PI3K inhibitor). Analysis of TUNEL staining indicated that dexmedetomidine improved LPS-induced neuronal apoptosis, which could be eradicated by AKT short hairpin RNA transfection, prazosin or yohimbine. Finally, dexmedetomidine ameliorated both the emotional and spatial cognitive disorders without alteration in locomotor activity. The present findings suggested that dexmedetomidine may protect the brain against SAE, and that the Hsp90/AKT pathway may be involved in this process.
\end{abstract}

Correspondence to: Dr Lijun Yin, Department of Anesthesiology, Tianjin Baodi Hospital, Baodi Clinical College of Tianjin Medical University, 8 Guangchuan Road, Tianjin 301800, P.R. China E-mail: yinlijun68@163.com

Key words: dexmedetomidine, sepsis-associated encephalopathy; Hsp90/AKT signaling, cecal ligation and peroration, lipopolysaccharide

\section{Introduction}

In intensive care units (ICUs), sepsis and its complications are the most common causes of mortality (1). Sepsis-associated encephalopathy (SAE) is clinically characterized by mental alterations that ranges from a transient brain damage to severe and irreversible encephalopathy (2). The mechanism of SAE is elusive but is considered to be multifactorial. A recent study has demonstrated that the mitochondrial respiratory chain and creatine kinase activities are involved in cecal ligation and perforation (CLP) rats (3). Additionally, the uncoupling of mitochondrial oxidative phosphorylation also takes place in the septic brain (4). These previous results suggested that mitochondrial dysfunction and neuronal apoptosis may be involved in the pathogenesis of SAE. Furthermore, SAE has also been reported to be associated with long-term learning and memory deficits (5).

Dexmedetomidine is a potent and highly selective $\alpha_{2}$-adrenergic receptor agonist with sedative, amnestic, sympatholytic and analgesic properties (6). Dexmedetomidine is reported to reduce intraoperative anesthetic requirements and offers good perioperative hemodynamic stability (7). In addition, dexmedetomidine decreases psychosis, shortens hospital stays and may improve cognitive dysfunctions (8). Therefore, dexmedetomidine is widely used for sedation, especially in ICUs (9). Dexmedetomidine has been shown to protect organs against stimuli in various models. For example, dexmedetomidine combined with ketamine reduces ventilator induced lung injury in endotoxemia rats (10). In addition, dexmedetomidine possess a protective effect on ischemia-reperfusion injury in heart, kidney, testis and intestine in various animal models (11-14). However, whether dexmedetomidine can improve neuronal apoptosis and protect brain against sepsis in SAE model remains to be elucidated.

The serine/threonine kinase AKT, also known as protein kinase $\mathrm{B}(\mathrm{PKB})$, is a pro-survival protein and involved in the regulation of cell apoptosis and caspase-3 activation (15). The heat-shock protein 90 (Hsp90) is a constitutively expressed stress protein located in the cytosol of eukaryotic cells, including neuronal cells (16). Hsp90 serves an essential role in controlling AKT functions. The formation of chaperone-substrate protein complex leads to a reduction in the binding between Hsp90 and AKT, resulting in AKT kinase inactivation (17). In myocardial cells, lipopolysaccharide (LPS) treatment contributes to the degradation of Hsp90 and AKT $(18,19)$. 
The Hsp90/AKT pathway is an important survival and antiapoptotic pathway in cells. For example, the cleavage of Hsp90 in the AKT/Hsp90 complex appears to be vital in the AKT/Hsp90 complex destabilization and in triggering the apoptotic cascade $(20,21)$. Dexmedetomidine has been shown to upregulate the AKT signaling pathway in a traumatic brain injury model (22). The present study explored the neuronal protective role of dexmedetomidine in an experimental septic model using CLP rats and LPS-treated primary neuronal cultures. In addition, the present study investigated the possible involvement of the Hsp90/AKT signaling pathway in this process.

\section{Materials and methods}

Animals and regents. A total of 146 adult male Sprague-Dawley rats (mean weight, $250 \mathrm{~g}$, weight range: 233-272 g; age, 8 weeks) were acquired from Tianjin Medical University. All studies performed on animals were approved by the local Institutional Animal Care and Use Committee (Tianjin Baodi Hospital, Baodi Clinical College Of Tianjin Medical University, Tianjin, China). All animal experiments complied with the ARRIVE guidelines (23) and the National Institutes of Health guide for the care and use of Laboratory animals (NIH Publications No. 8023, revised 1978) (24). The animals were housed at $22 \pm 1^{\circ} \mathrm{C}$ with $50-70 \%$ humidity, and maintained on a 12-h light/dark cycle throughout the experiments. Every effort was made to minimize suffering and the number of animals used in the present study. All animals were given food and water ad libitum. DMEM, 10\% FBS, poly-L-lysine, Neurobasal media, L-glutamine and B27 were purchased from Gibco (Thermo Fisher Scientific, Inc.). Trypsin-EDTA, Cell Counting Kit-8 (CCK-8), lactate dehydrogenase (LDH), pentobarbital, prazosin hydrochloride, yohimbine hydrochloride and wortmannin were purchased from Sigma-Aldrich (Merck KGaA). TUNEL staining reaction solution was obtained from Roche Applied Science, 17-AAG was purchased from Tocris BioScience, and Rhodamine (TRITC)-Streptavidin from Jackson ImmunoResearch Laboratories, Inc. Dexmedetomidine hydrochloride injection was acquired from Jiangsu Hengrui Medicine Co., Ltd.

Hippocampal neuronal cultures and cell transfection. Primary hippocampal neuronal cells were isolated from embryonic day 18 Sprague-Dawley rats as previously described (25). In brief, the fetuses were removed from pregnant rats (a total of 18 rats provided by Nanjing University, weight $455-529 \mathrm{~g}$, aged 10 weeks) euthanized by $\mathrm{CO}_{2}$ anoxia at concentrations of $100 \%$. Hippocampal tissues were dissociated with $0.25 \%$ Trypsin-EDTA. The digestion was terminated using DMEM combined with $10 \%$ FBS Neuronal cells were cultured in96-well plates (for LDH and CCK-8 detection), coverslips (for TUNEL-staining) or dishes (for western blotting) that were precoated with poly-L-lysine. Neurons were fed with Neurobasal media supplemented with $500 \mu \mathrm{M}$ of L-glutamine and $2 \%$ of B27, and maintained in a humidified atmosphere of $5 \% \mathrm{CO}_{2}$ and $95 \% \mathrm{O}_{2}$ at $37^{\circ} \mathrm{C}$. After 3 days of incubation, half of the medium was replaced with fresh medium. A short hairpin RNA (shRNA) was used to downregulate AKT, and the
shRNA sequence (starting point 221-239) was cloned into a lentiviral expression vector. The concentration of shRNA transfected was $1 \mu \mathrm{g} / \mathrm{ml}$ according to the instruction of the manufacturer. The AKT shRNA sequence was the follow: Sense, 5'-GATCCGTCAGCTGATGAAGACAGATTCAAG ACGTCTGTCTTCATCAGCTGACTTTTTTGTCGACA-3'; anti-sense, 5'-AGCTTGTCGACAAAAAAGTCAGCTGAT GAAGACAGACGTCTTGAATCTGTCTTCATCAGCTGA CG-3'. Recombinant AKT shRNA lentiviral vector and negative control lentiviral vector were diluted into $10^{8} \mathrm{TU} / \mathrm{ml}$ with the high-efficiency lentivirus transfection enhancement solution (Invitrogen, Thermo Fisher Scientific, Inc.). At 14 days in vitro, neuronal cells were infected with the recombinant AKT shRNA lentiviruses using the Lipofectamine $2000^{\circledR}$ reagent (Invitrogen, Thermo Fisher Scientific, Inc.). The shRNA oligonucleotides were synthesized by Shanghai Sangon Biological Engineering Technology \& Services Co., Ltd. (Shanghai, China). Different dose of dexmedetomidine $(0.1,0.5$ or $1.0 \mu \mathrm{M})$ or LPS $(1 \mu \mathrm{g} / \mathrm{ml})$ were used to treat cells $48 \mathrm{~h}$ after cell transfection.

Neuronal survival evaluation. The neuronal survival was evaluated by measuring the level of LDH released into the culture media and this evaluation was confirmed by CCK- 8 assay. These experiments were conducted $24 \mathrm{~h}$ after the drug treatments. Briefly, for the LDH assay, $50 \mu \mathrm{l}$ neurobasal medium was mixed with equal volume of substrate maintaining at $37^{\circ} \mathrm{C}$. After incubation for $30 \mathrm{~min}, 50 \mu \mathrm{l}$ of stop solution was then added. The absorbance at $490 \mathrm{~nm}$ was recorded using a microtiter plate reader (Quant; Bio-Tek Instruments Inc.). For the CCK-8 assay, $10 \mu 1$ of CCK-8 solution was incubated with cells at $37^{\circ} \mathrm{C}$ for $2.5 \mathrm{~h}$. Absorbance at $450 \mathrm{~nm}$ was then measured.

Cecal ligation and perforation surgery. An animal model of SAE was established by cecal ligation and perforation (CLP) surgery as previously described (26). Briefly, after intraperitoneally injecting with $50 \mathrm{mg} / \mathrm{kg}$ of pentobarbital for anesthesia, a midline incision $(\sim 3 \mathrm{~cm})$ was performed to allow the exposure of the cecum. The cecum was isolated carefully, and ligated tightly below the ileocecal junction. The cecum was punctured twice with a 16-gauge needle, and gently compressed to remove a small amount of cecal contents. The cecum was then put back into the abdominal cavity. Finally, the abdominal wall was closed. For sham-operated animals, which were used as control, the rats underwent the same procedure but without ligation and perforation. After surgery, the CLP rats were subcutaneously (s.c.) injected with a solution containing saline $(50 \mathrm{ml} / \mathrm{kg})$ immediately and $12 \mathrm{~h}$ after CLP performance. In addition, the rats were injected with $30 \mathrm{mg} / \mathrm{kg}$ of ceftriaxone and $25 \mathrm{mg} / \mathrm{kg}$ of clindamycin every $6 \mathrm{~h}$ for consecutive 3 days. The sham-operated group received only saline $(50 \mathrm{ml} / \mathrm{kg}$ immediately and $12 \mathrm{~h}$ after CLP surgery). During their recovery from sepsis, all animals were observed after CLP to detect signs of infection (including piloerection, lethargy, tachypnea and weight loss). If a rat demonstrated constant lethargic sleep or/and did not take food or water for 48 consecutive $\mathrm{h}$, the rat was evaluated by two researchers. If both researchers confirmed that the rat would succumb to sepsis, the rat was euthanized by being deeply anesthetized 
with sodium pentobarbital $(120 \mathrm{mg} / \mathrm{kg}$, i.p.) followed by cervical dislocation. The researchers in the present study used utmost efforts to minimize the quantity and the suffering of all experimental animals. Survival rateswere $100 \%$ in the sham group and approximately $36 \%$ in the sepsis group, which is in accordance with a previous study (27).

Drug administration. Following surgery, all animals were administrated with isotonic saline solution $(50 \mathrm{ml} / \mathrm{kg} \mathrm{s.c.}$ ) and antibiotics (ceftriaxone, $30 \mathrm{mg} / \mathrm{kg}$; clindamycin, $25 \mathrm{mg} / \mathrm{kg}$ ) immediately after and $12 \mathrm{~h}$ after CLP surgery. The sham-surgery rats received the same volume of isotonic saline solution without antibiotics. At $6 \mathrm{~h}$ after surgery, the CLP rats were intraperitoneally injected with different doses of dexmedetomidine $(1,5$ and $10 \mu \mathrm{g} / \mathrm{kg}$ ) every $12 \mathrm{~h}$ consecutively for a week. The control rats were administrated with the same volume of vehicle. 17-AAG (dose, $10 \mathrm{mg} / \mathrm{kg}$ ), wortmannin (dose, $50 \mathrm{mg} / \mathrm{kg}$ ) or same volume of vehicle was also injected intraperitoneally once a day for 1 week. The whole hippocampal tissues were subjected to western blot analysis 1 week after surgery.

Western blot analysis. Whole hippocampal tissues and primary neuronal cultures were collected and subjected to lysis buffer (Tris-HCl: 50 mM, pH 7.4; NP-40: 1\%; Sodium deoxycholate: 0.25\%; NaCl: $150 \mathrm{mM}$; EDTA: $1 \mathrm{mM}$; PMSF: $1 \mathrm{mM}$; Aprotinin, leupeptin, pepstatin: $1 \mu \mathrm{g} / \mathrm{ml}$ each; Na3VO4: $1 \mathrm{mM}$; NaF: $1 \mathrm{mM}$ ). Bradford Protein Assay (Bio-Rad Laboratories, Inc.) was used to quantify the protein concentration of samples. Lysates $(30 \mu \mathrm{g})$ were loaded and separated by $10-15 \%$ sodium dodecyl sulfate-polyacrylamide gel electrophoresis (SDS-PAGE) (Beyotime Institute of Biotechnology) and transferred them onto PVDF membranes (Beyotime Institute of Biotechnology). The membranes were blocked by $5 \%$ skimmed milk for $1 \mathrm{~h}$ at room temperature, and then incubated overnight at $4^{\circ} \mathrm{C}$ with the appropriate primary antibodies. After three washes with PBS-T (0.1\% Tween-20), membranes were incubated with horseradish peroxidase (HRP)-conjugated appropriate secondary antibodies for $2 \mathrm{~h}$ at room temperature. Polyclonal rabbit anti-cleaved caspase-3 (C9598; 1:2,000), polyclonal rabbit anti-Bcl-2 (SAB4500005; 1:1,500) and monoclonal mouse anti-Hsp90 (SAB1305541; 1:2,000) were from Sigma-Aldrich (Merck KGaA). Polyclonal rabbit anti-AKT (sc-55523; 1:1,000) and anti-phosphorylated(p)-AKT-Ser 473 (sc-24500; 1:1,000) were obtained from Santa Cruz Biotechnology, Inc. Monoclonal antibody anti-Actin (58169; 1:1,000) was purchased from Cell Signaling Technology, Inc. Goat anti-rabbit HRP-conjugated IgG (A0208; 1:1,000) and goat anti-mouse HRP-conjugated IgG (A0216; 1:1,000) were from Beyotime Institute of Biotechnology. The membranes were washed and processed with ECL kit (Gibco; Thermo Fisher Scientific, Inc.) and then read digitally with Image ReaderLAS-4000 (FujiFilm Life Science). The bands were quantified using Multi Gauge version 3.0 software (FujiFilm Life Science).

TUNEL assay. TUNEL assay was used to determine neuronal DNA damage. Briefly, the neuronal cells on coverslips were permeabilized with $0.01 \%$ Triton X-100 (Sigma-Aldrich; Merck KGaA) in PBS containing $1 \%$ sodium citrate. The TUNEL reaction mixture was added into and co-incubated with the neuronal cultures at $37^{\circ} \mathrm{C}$ for $30 \mathrm{~min}$. Following washing with PBS, the coverslips were then incubated with Rhodamine (TRITC)-Streptavidin at $37^{\circ} \mathrm{C}$ for $1 \mathrm{~h}$. Hoechst 33342 (Beyotime Institute of Biotechnology) was used for nuclei staining. In brief, $1 \%$ of Hoechst 33342 was added on coverslips and incubated for $5 \mathrm{~min}$ at room temperature according to the instructions of the manufacturer. Three separate cultures and 20 fields (magnification, x40) per culture were examined in each group. Immunofluorescence of neuronal cells was captured and analyzed by a laser scanning confocal microscope (Zeiss AG). The TUNEL-positive cells were considered to be apoptotic.

\section{Behavioral studies}

Open-field test. The behavior study was performed 2 weeks after surgery. The open-field test was used to detect the possible locomotor activity deficits. In brief, each animal was released in the center of the arena, and allowed to walk freely for $10 \mathrm{~min}$. At the end of each test, the arena was carefully cleaned with alcohol to avoid the presence of olfactory cues. The total distance traveled in $10 \mathrm{~min}$ was measured, and considered as locomotor activity of the experimental animal.

Morris water maze. A Morris water maze (Shanghai Bio-will Co.) was used to evaluate spatial cognitive functions as previously described (28). Place trials were performed to determine the spatial learning capability. All rats received two trails each day for consecutive three days. The rats were individually placed into the pool facing the sidewall. They were allowed $1 \mathrm{~min}$ to find the platform that was $2.5 \mathrm{~cm}$ below the water surface. The time to reach the platform (latency) and the swimming speed were recorded. Probe test was carried out to determine capability of memory retention. After the last place trial, the platform was removed. All rats were allowed to swim for 1 min starting from the same quadrant. The number of crossings over the platform and the swimming time spent in each quadrant were recorded. All swimming paths were tracked by a video monitoring system (Coulbourn Instruments, LLC).

Fear conditioning. The contextual and cued fear conditioning experiment was performed to examine the capability of emotional learning and memory as previously described (29). The fear conditioning consisted of a 5-min free exploration period followed by three conditioned stimulus and unconditioned stimulus pairings separated by $1 \mathrm{~min}$ each. At the end of the conditioned stimulus presentation (80 db noise for $20 \mathrm{sec}$ ), $1 \mathrm{~mA}$ of foot shock was delivered for $1 \mathrm{sec}$ as unconditioned stimulus. The contextual test was carried out in the conditioning chamber for $5 \mathrm{~min}$ in the absence of noise at $24 \mathrm{~h}$ after conditioning. The cued test was conducted $48 \mathrm{~h}$ after conditioning by presentation of a cue $(80 \mathrm{db}$ noise for $3 \mathrm{~min}$ ) in an alternative context with distinct visual and tactile cues. The context was changed in several ways to test conditioned fear of the tone CS in the absence of contextual cues associated with shock. First, the walls and floor of chamber were covered by black and white plastic inserts. In addition, the chamber was scented with limonene, and the experimenter wore a different style of gloves. Furthermore, all rats were kept in a different holding room before testing. The rate of freezing time was recorded. 

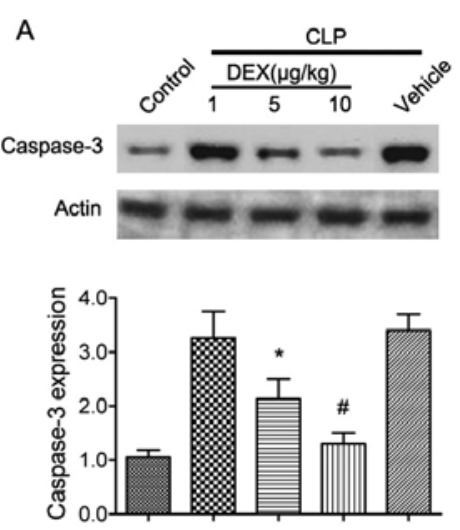

B
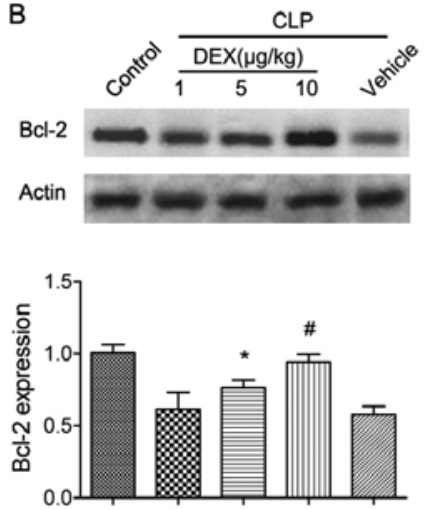
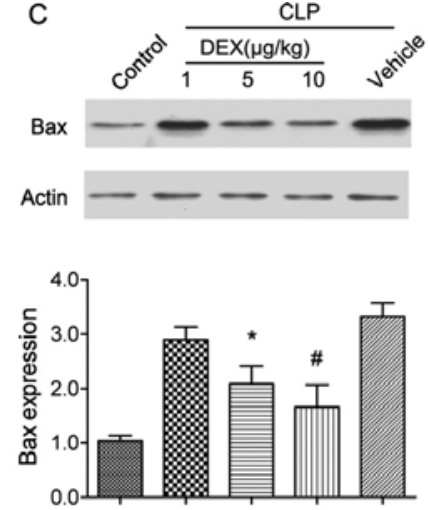

D

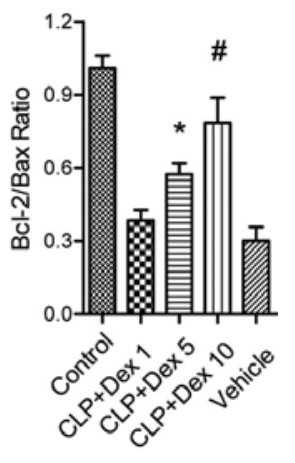

Figure 1. Administration of dexmedetomidine alleviates neuronal apoptosis in CLP rats. The CLP rats were injected with different doses of dexmedetomidine $(1,5$ or $10 \mu \mathrm{g} / \mathrm{kg})$ or the same volume of vehicle. The expressions of (A) cleaved caspase-3, (B) Bcl-2and (C) Bax were detected by western blotting. The histogram represents the relative expressions of cleaved caspase-3, Bcl-2 and Bax. Actin was re-probed as a loading control. (D) The histogram indicates Bcl-2/Bax ratio. Data are expressed as mean $\pm \mathrm{SEM},(\mathrm{n}=6-8)$. Statistical significance was determined by ${ }^{*} \mathrm{P}<0.05$ and ${ }^{\#} \mathrm{P}<0.01$ vs. vehicle group. CLP, cecal ligation and perforation; DEX, dexmedetomidine.

Statistical analysis. All data are expressed as mean and standard error of the mean. Normal distribution of the residuals was assessed by the Shapiro-Wilk test. Comparisons among multiple groups were analyzed using one-way ANOVA followed by Tukey's multiple comparison test. For Morris water maze analysis, two-way ANOVA (treatment as between-groups and time as repeated measures factors) followed by Bonferroni multiple comparison testing was used. Data analysis was generated using GraphPad Prism 5.0 (GraphPad Software, Inc.). $\mathrm{P}<0.05$ was considered to indicate a statistically significant difference.

\section{Results}

Administration of dexmedetomidine attenuates neuronal apoptosis in CLP rats. Mitochondria-mediated neuronal apoptosis has been reported to be involved in experimental sepsis (30). The protein level of cleaved caspase-3 and Bcl-2 were detected to evaluate the neuronal protective role of dexmedetomidine in CLP rats. Following CLP surgery and dexmedetomidine treatment $(1,5$ and $10 \mu \mathrm{g} / \mathrm{kg})$, the hippocampal tissues were harvested for western blot analysis. It was found that CLP treatment significantly increased the expression of cleaved caspase- 3 and $\operatorname{Bax}(\mathrm{P}<0.05$; Fig. 1A and $\mathrm{C})$, but decreased Bcl-2 ( $\mathrm{P}<0.05$; Fig. 1B). Application of 5 or $10 \mu \mathrm{g} / \mathrm{kg}$ dexmedetomidine significantly restored the alterations of cleaved caspase-3, Bcl-2 and $\mathrm{Bax}(\mathrm{P}<0.05$; Fig. 1A-C). In addition, application of dexmedetomidine (5 or $10 \mu \mathrm{g} / \mathrm{kg}$ ) also significantly increased the $\mathrm{Bcl}-2 /$ Bax ratio $(\mathrm{P}<0.05$; Fig. 1D). The present results suggested that dexmedetomidine may protect brain against sepsis by suppressing the intrinsic cell apoptosis signaling pathway.

Dexmedetomidine increases neuronal survival in LPS treated hippocampal cultures. To investigate the possible neuronal protective effects of dexmedetomidine against endotoxemia, the primary cultured hippocampal neurons (14 DIV) were co-incubated with LPS $(1 \mu \mathrm{g} / \mathrm{ml})$ and different doses of dexmedetomidine $(0.1,0.5$ or $1 \mu \mathrm{M})$ for $24 \mathrm{~h}$. The neuronal injury was quantitatively evaluated through the measurement of LDH activity in the medium. Analysis of LDH assay suggested that LPS treatment significantly increased LDH levels $(273 \pm 29 \%, \mathrm{P}<0.05$; Fig. $2 \mathrm{~A})$, which were statistically decreased following treatment with 0.5 or $1 \mu \mathrm{M}$ of dexmedetomidine (163 \pm 11 and $127 \pm 18 \%$, respectively, $\mathrm{P}<0.05$; Fig. $2 \mathrm{~A})$. Administration of dexmedetomidine $(1 \mu \mathrm{M})$ alone had no effects on neuronal survival ( $\mathrm{P}>0.05$; Fig. 2A). These results were confirmed by CCK- 8 assay, which showed similar results (Fig. 2B). Therefore, $1 \mu \mathrm{M}$ of dexmedetomidine was selected for the following in vitro experiments. The neuronal protective role of dexmedetomidine was also morphologically assessed by TUNEL-staining. Analysis of data indicated that $1 \mu \mathrm{M}$ of dexmedetomidine significantly improved the neuronal apoptosis induced by LPS treatment $(\mathrm{P}<0.05$; Fig. 3A and $\mathrm{B})$.

Hsp90/AKT signaling pathway is involved in the neuronal protective effects of dexmedetomidine. Several previous studies have demonstrated that AKT can be phosphorylated by Hsp90 at the Thr 308 site, and serves an essential role in regulating neuronal fate $(15,16)$. The involvement of the Hsp90/AKT signaling pathway in the neuronal protective effects of dexmedetomidine in rat models of CLP was therefore examined. In the present study, the expressions of Hsp90 and p-AKT Thr 308 were significantly downregulated in CLP rats $(\mathrm{P}<0.05$; Fig. 4). Administration of dexmedetomidine significantly ameliorated the inhibition of Hsp90 and p-AKT Thr 308 ( $\mathrm{P}<0.05$; Fig. 4A and B).

To further determine the involvement of the Hsp90/AKT signaling pathway, 17-AAG (a Hsp90 inhibitor) or wortmannin (a PI3K inhibitor) was injected in CLP rats. It was found that the neuronal protective effects of dexmedetomidine were abolished by 17-AAG or wortmannin $(\mathrm{P}<0.05$; Fig. 5). To confirm these results, AKT shRNA was transfected into hippocampal neurons for $48 \mathrm{~h}$. The transfection efficiency was shown in Fig. 6B. It was found that the anti-apoptotic role of dexmedetomidine was also reversed by $17-A G G$, PI3K inhibitor wortmannin, or AKT shRNA transfection, as assessed by TUNEL-assay ( $\mathrm{P}<0.05$; Fig. 6A).

Dexmedetomidine is an $\alpha_{2}$-adrenergic receptor agonist (31), so the hypothesis that the neuronal protective role of dexmedetomidine could be abolished by $\alpha_{2}$-adrenergic receptor antagonist was then tested. Prazosin $(0.5 \mu \mathrm{M})$ or yohimbine 
A

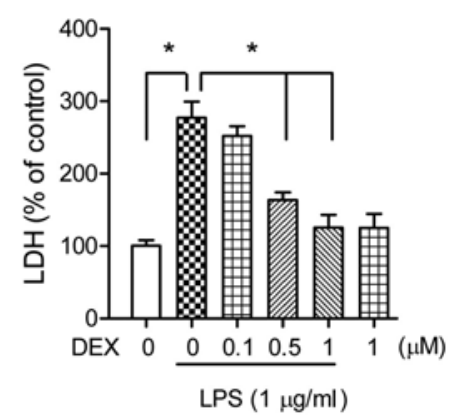

B

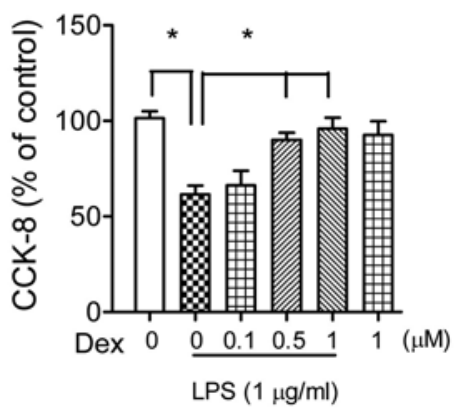

Figure 2. Dexmedetomidine increases cell viability in LPS treated neuronal cultures. The neuronal cultures (14 DIV) were co-incubated with LPS $(1 \mu \mathrm{g} / \mathrm{ml})$, LPS combined with different doses of dexmedetomidine $(0.1,0.5$ or $1 \mu \mathrm{M})$ or dexmedetomidine $(1 \mu \mathrm{M})$ alone for $24 \mathrm{~h}$. Cell viability was determined by measurement of (A) LDH release and (B) CCK-8 reduction. Statistical significance was determined by ${ }^{*} \mathrm{P}<0.05$. $(\mathrm{n}=7)$. Data are expressed as mean \pm SEM. LPS, lipopolysaccharide; DEX, dexmedetomidine; $\mathrm{LDH}$, lactate dehydrogenase.

A
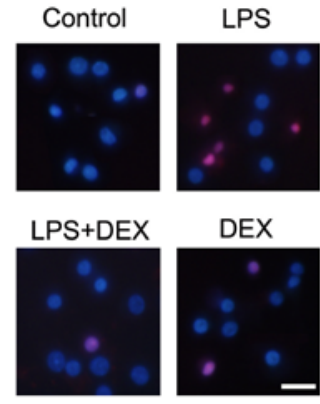

DEX

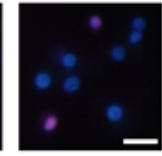

B

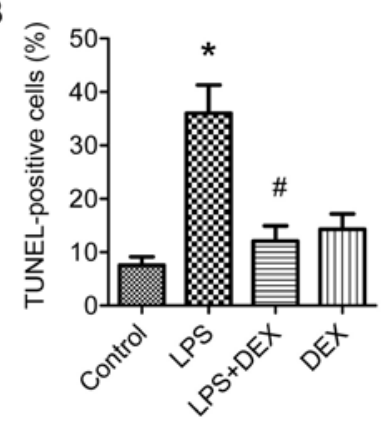

Figure 3. Dexmedetomidine inhibits neuronal apoptosis induced by LPS treatment. (A) Primary hippocampal neuronal cultures were co-incubated with LPS $(1 \mu \mathrm{g} / \mathrm{ml})$, or combined with dexmedetomidine $(1 \mu \mathrm{M})$ or dexmedetomidine $(1 \mu \mathrm{M})$ alone for $24 \mathrm{~h}$. The neurons were stained with TUNEL reaction solution. Hoechst 33342 was used for nuclei-staining. Immunofluorescence of neuronal cells was captured by a laser scanning confocal microscope. The TUNEL-positive cells were considered to be apoptotic (scale bar=50 $\mu \mathrm{m}$ ). (B) Separate cultures (3) and 20 fields per culture were examined in each group. The histogram represents the percentage of TUNEL-positive cells ${ }^{*} \mathrm{P}<0.05$ vs. control group; ${ }^{\#} \mathrm{P}<0.05$ vs. LPS group. Data are expressed as mean \pm SEM, $(n=8)$. LPS, lipopolysaccharide; DEX, dexmedetomidine.
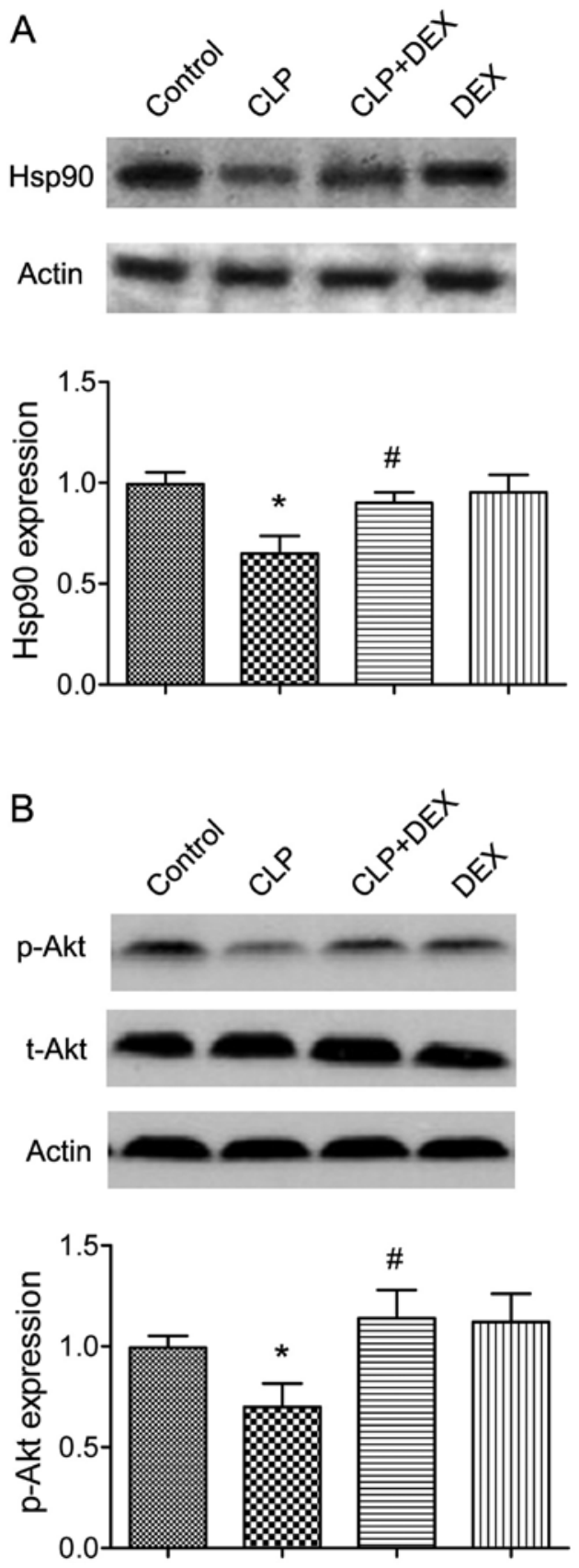

Figure 4. The Hsp90/AKT signaling pathway was inhibited in CLP rat model. Sham or CLP surgery rats were administrated with dexmedetomidine $(10 \mu \mathrm{g} / \mathrm{kg})$ or vehicle. The hippocampal tissues were harvested and subjected to western blot analysis to detect the expression of (A) Hsp90 and (B) total AKT and phospho-AKT Thr 308. Actin was probed as a loading control. The histogram represents the relative expressions of $\mathrm{Hsp} 90$ and phospho-AKT Thr 308. Data are expressed as mean \pm SEM, $(n=6)$. Difference was determined by ${ }^{*} \mathrm{P}<0.05$ vs. control group, and ${ }^{\#} \mathrm{P}<0.05$ vs. CLP group. CLP, cecal ligation and perforation; DEX, dexmedetomidine.

$(0.3 \mu \mathrm{M})$ was coincubated with LPS-treated neuronal cultures. It was found that either prazosin or yohimbine had the capability to revert the anti-apoptotic effects of dexmedetomidine $(\mathrm{P}<0.05$; Fig. 6A).

Dexmedetomidine improves the spatial and emotional disorders in CLP rats. The behavior study was performed 2 weeks after CLP surgery, according to the protocol. In the open-field test, it was found that there was no difference among 
A
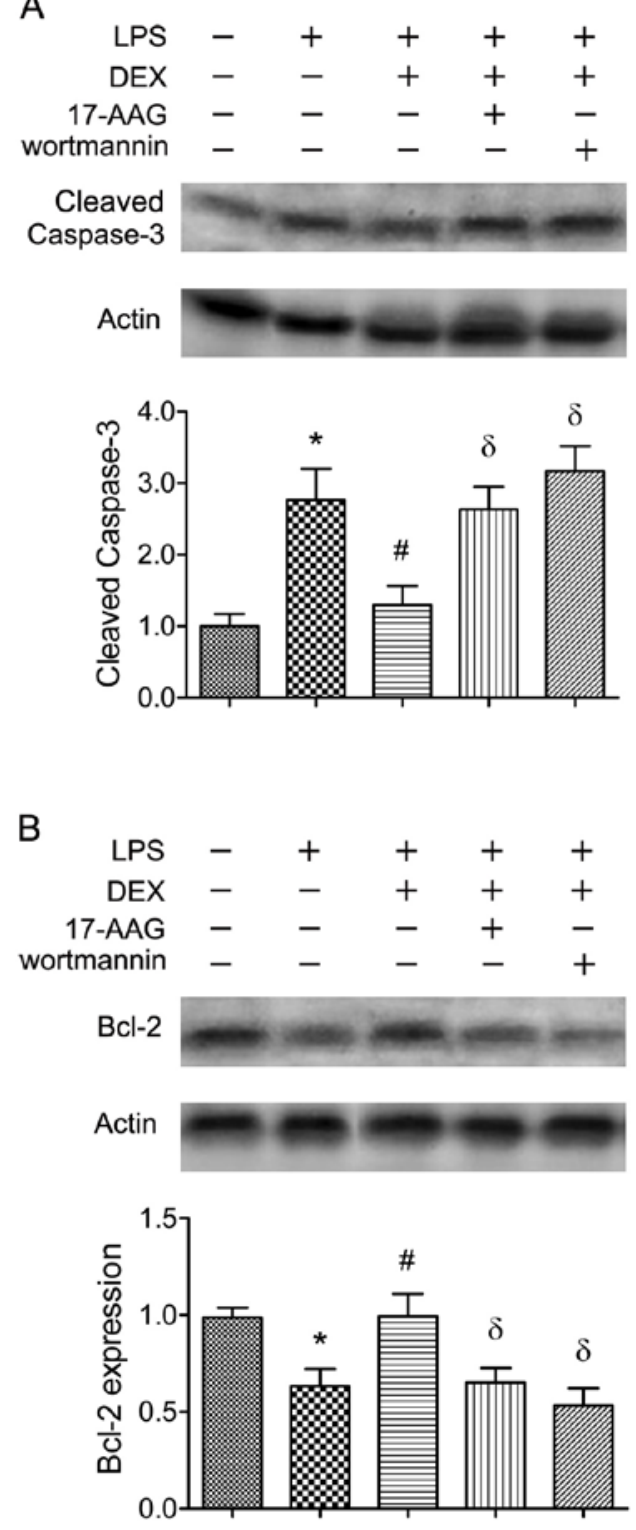

Figure 5. Dexmedetomidine protects brain against sepsis through Hsp90/AKT signaling pathway. The CLP rats were administered dexmedetomidine or further treated with 17-AAG $(10 \mathrm{mg} / \mathrm{kg}$, i.p.), PI3K inhibitor wortmannin $(50 \mathrm{mg} / \mathrm{kg}$, i.p.) or vehicle. Hippocampal tissues were harvested for evaluating the expressions of (A) Cleaved caspase-3 and (B) Bcl-2 by western blot analysis. The histograms show the relative expressions of Cleaved caspase-3 and Bcl-2. " $\mathrm{P}<0.05$ vs. control group, ${ }^{*} \mathrm{P}<0.05$ vs. LPS group, ${ }^{\delta} \mathrm{P}<0.05$ vs. LPS + DEX group, $(\mathrm{n}=7)$. CLP, cecal ligation and perforation; DEX, dexmedetomidine; LPS, lipopolysaccharide.

groups as for the traveled distance ( $\mathrm{P}>0.05$; Fig. $7 \mathrm{~A})$. For the place trials, CLP rats were found to spend significant more time to find the submerged platform at trial 3 day compared with control rats $(\mathrm{P}<0.05$; Fig. 7B). The rats in the CLP + DEX group were compared with the control group in terms of latency to find the submerged platform ( $\mathrm{P}>0.05$; Fig. 7B). There were no statistical differences in terms of average swimming speeds ( $\mathrm{P}>0.05$; Fig. 7C), suggesting that dexmedetomidine did not alter the locomotor activity. As for the probe test in Morris water maze, the CLP rats had fewer crossings compared with those of control rats $(\mathrm{P}<0.05$; Fig. 7D). Rats of CLP +
A
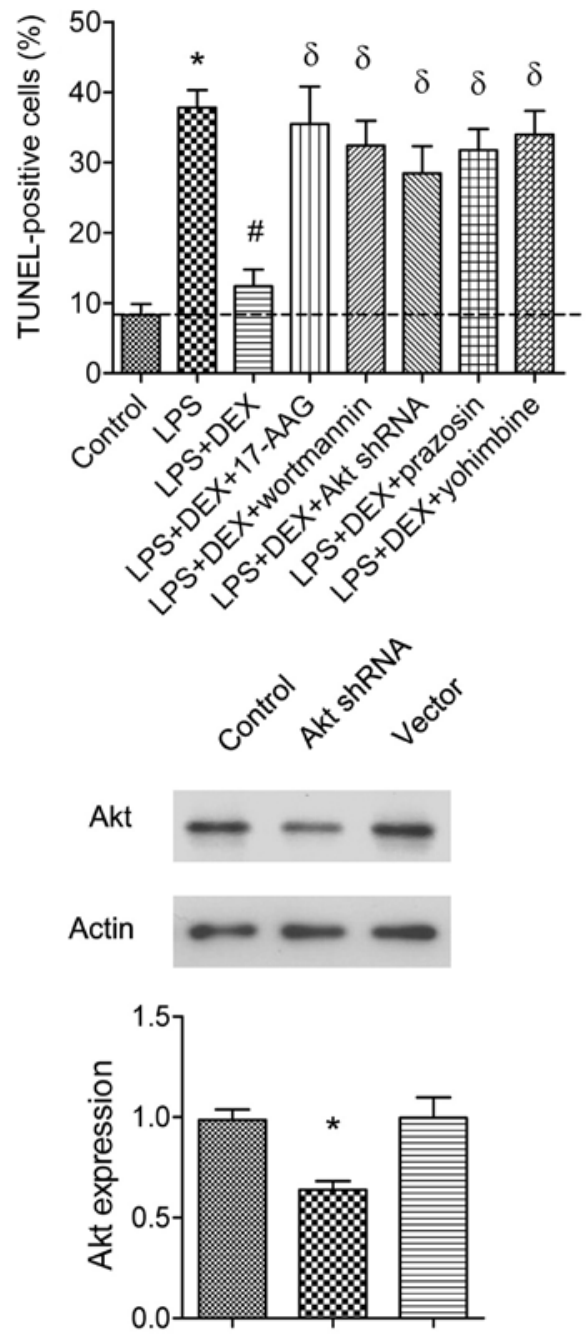

Figure 6. Assessment of apoptotic cells by TUNEL assay in primary hippocampal neuronal cultures. (A) Neuronal cultures were co-incubated with LPS $(1 \mu \mathrm{g} / \mathrm{ml})$, or combined with dexmedetomidine $(1 \mu \mathrm{M})$, 17-AAG $(1 \mu \mathrm{M})$, wortmannin $(5 \mu \mathrm{M})$, prazosin $(0.5 \mu \mathrm{M})$ or yohimbine $(0.3 \mu \mathrm{M})$ as indicated. Apoptotic neurons were evaluated by TUNEL assay. The histogram shows the percentage of TUNEL-positive cells. ${ }^{~} \mathrm{P}<0.05$ vs. control group, ${ }^{~} \mathrm{P}<0.05$ vs. LPS group, ${ }^{\delta} \mathrm{P}<0.05$ vs. LPS + DEX group, $(\mathrm{n}=8)$. (B) Neuronal cultures were transfected with AKT shRNA or vector as control. The AKT protein expression was detected by western blot analysis. ${ }^{*} \mathrm{P}<0.05$ vs. control group, $(\mathrm{n}=5)$. LPS, lipopolysaccharide; DEX, dexmedetomidine; shRNA, short hairpin RNA.

DEX group had significantly more crossings compared with rats of CLP group ( $\mathrm{P}<0.05$; Fig. 7D). In addition, CLP rats spent significantly less time searching the target quadrant than those of control group ( $\mathrm{P}<0.05$; Fig. 7E). Rats administered dexmedetomidine spent significantly more time in the target quadrant than CLP animals $(\mathrm{P}<0.05$; Fig. 7E). In the fear-conditioning test, it was found that the freezing time of CLP group was significantly reduced compared with control rats both in the 24-h context and 48 -h cue tests $(\mathrm{P}<0.05$; Fig. 7F and G). However, the freezing time in rats treated with dexmedetomidine was significantly increased compared with the rats of the CLP group in the 24-h context and 48-h cue tests $(\mathrm{P}<0.05$; Fig. 7F and G). Collectively, the behavioral results of the present study suggested that dexmedetomidine ameliorated both the spatial and emotional deficits induced by systemic sepsis. 

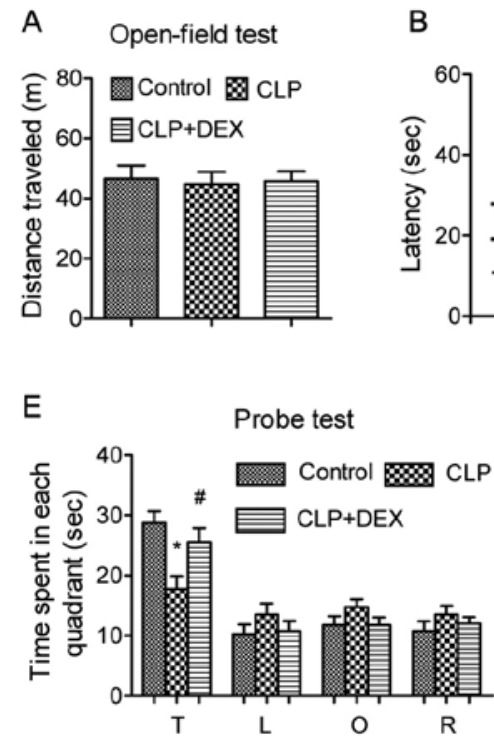

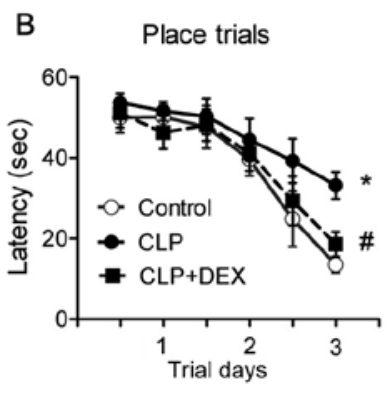

$\mathrm{F}$
C

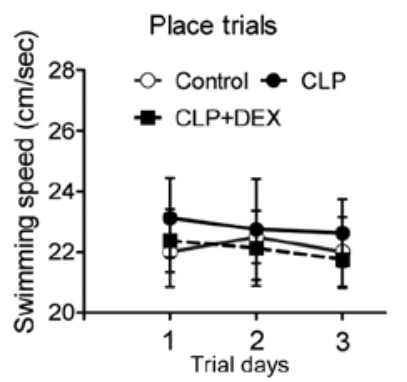

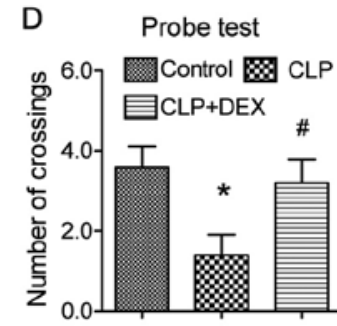

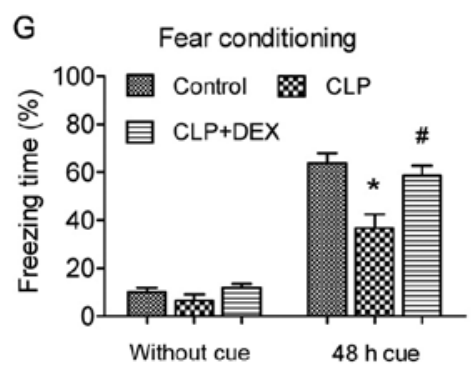

Figure 7. Dexmedetomidine ameliorates the spatial and emotional cognitive disorders in CLP rats. The behavior study was performed 2 weeks after CLP surgery. The Open-field test, Morris water maze and fear-conditioning test were performed to examine the locomotor activity, spatial and emotional cognitive disorders respectively. (A) The histogram shows the total distance of each group rats traveled in 10 min. (B) In place trials of Morris water maze, all rats received two blocks of trails (two trials per block and $2 \mathrm{~h}$ rest between blocks) each day for consecutive 3 days. Latency was defined as the time to reach the submerged platform. (C) Each swimming speed of rat was recorded, and the average in place trials of Morris water maze was calculated. (D) The histogram represents the number of crossings of each group in probe test of Morris water maze. (E) In the probe test, the platform was removed and all rats commenced to swim $(1 \mathrm{~min})$ in the same quadrant. The histogram represents the percentage of swimming time spent in each quadrant. The fear conditioning consisted of a 5-min free exploration period followed by three conditioned stimulus ( $80 \mathrm{db}$ noise for $20 \mathrm{sec}$ ) and unconditioned stimulus ( $1 \mathrm{~mA}$ for $1 \mathrm{sec})$ pairings. (F) The contextual test was carried out in the conditioning chamber for $5 \mathrm{~min}$ in the absence of noise at $24 \mathrm{~h}$ after conditioning. (G) The cued test was conducted $48 \mathrm{~h}$ after conditioning by presentation of a cue $(80 \mathrm{db}$ noise for $3 \mathrm{~min})$ in an alternative context with distinct visual and tactile cues. The rate of freezing time was recorded. All data are expressed as mean \pm SEM. The experimental rats were 17, 21 and 19 in control, CLP and CLP+DEX group respectively. Statistical significance was determined by ${ }^{*} \mathrm{P}<0.05$ vs. control group, and ${ }^{\#} \mathrm{P}<0.05$ vs. CLP group. CLP, cecal ligation and perforation; DEX, dexmedetomidine; $\mathrm{T}$, target; L, left; O, opposite; R, right.

\section{Discussion}

SAE is the neurological manifestation of sepsis and has been reported to occur in $9-71 \%$ of patients with sepsis (32). Patients with SAE have a higher mortality rate compared with those without brain complications (33). Although SAE could be caused by multiple factors including inflammation, reduced cerebral blood flow, disruption of the blood-brain barrier, cell injury of endothelium and cerebral edema $(34,35)$, the molecular mechanism underlying this process remains to be elucidated. The present study demonstrated that administration of dexmedetomidine decreased neuronal apoptosis and enhanced cell viability in vitro and in vivo. In addition, it was also found that dexmedetomidine markedly improved both the spatial and emotional dysfunctions in CLP rats. Furthermore, the present study provided evidence that Hsp90/AKT pathway may be involved in the neuronal protective effects of dexmedetomidine in septic model. To the best of the authors' knowledge, this is the first study demonstrating the anti-apoptotic role of consecutive dexmedetomidine exposure (1 week) in SAE model.

Dexmedetomidine exposure is an off-label therapy in clinical contexts. Accumulating literature has shown the neuronal protective role of dexmedetomidine in brain impairment $(36,37)$. The current study demonstrated that dexmedetomidine improved neuronal apoptosis induced by systemic sepsis. Of note, the sedative property of dexmedetomidine is dose-dependent. A recent study demonstrated that $100 \%$ of rats given $10 \mu \mathrm{g} / \mathrm{kg}$ dexmedetomidine were still able to right themselves with in 2 sec. However, only $33 \%$ of rats were still able to right themselves at $30 \mathrm{~min}$ when the concentration increased to $25 \mu \mathrm{g} / \mathrm{kg}$ (38). In the present study, consecutive dexmedetomidine exposure $(10 \mu \mathrm{g} / \mathrm{kg}$, i.p.) up to 1 week was chosen for the rats of the CLP model. In recent years, dexmedetomidine has been increasingly used for consecutive sedation of ICU patients (39). This prolonged dexmedetomidine exposure during recovering from systemic sepsis was adopted in order to simulate an actual clinical practice in ICUs. Prolonged dexmedetomidine exposure (average 2-3 weeks) has been investigated to be safe in human study (38).

AKT is a central node in cell signaling downstream of cytokines, growth factors, and other cellular stimuli (40). Aberrant loss or gain of AKT activation underlies the pathophysiological properties of a variety of complex diseases, including neuronal apoptosis and survival (41). The present study identified that AKT activity inhibition or AKT shRNA transfection reversed the neuronal protective effects of dexmedetomidine, indicating that AKT signaling serves an essential role in the neuroprotection of dexmedetomidine against sepsis. In addition, AKT pathway has also been reported to be involved in the dexmedetomidine's biological effects encountering transient focal ischemia/reperfusion injury (24), isoflurane induced neuronal apoptosis (42), neuropathic pain (43) and LPS-induced acute lung injury (10). Activation of $\alpha 2$-adrenergic receptor is associated with increased AKT signaling in lung apoptosis (44), 
heart ischemia/reperfusion injury (45) and arachidonic acid metabolism by cytochrome P450-dependent epoxygenase (46). Taken together, these previous studies indicate that AKT signaling pathway may serve a critical function in molecular mechanisms of the $\alpha 2$-adrenergic receptor.

Caspase- 3 and $\mathrm{Bcl}-2$ are important factors in intrinsic neuronal apoptosis. The present study demonstrated that dexmedetomidine treatment enhanced Bcl-2, but decreased caspase-3 expression, suggesting that dexmedetomidine protects the brain against sepsis mainly through inhibiting mitochondria-mediated neuronal apoptosis. Bad, as a proapoptotic Bcl-2 family member, can also be phosphorylated by AKT, and allow Bcl-xl to bind to the pro-apoptotic protein Bax $(47,48)$. Hypophosphorylated Bad interacts with the pro-survival Bcl-2 family proteins, which frees Bak and Bax to induce apoptosis at the mitochondria (49). In addition to Bcl-2, AKT activation controls cell survival through phosphorylation of downstream effector proteins, such as glycogen synthase kinase $3 \beta$, Bad, Bid, Bax, caspase-9, and possibly apoptosis inducing factor (50). Therefore, further studies are required to identify the involvement of other Bcl-2 family members and AKT downstream signaling in a dexmedetomidine-exposed SAE model.

In the present study, the improvements of spatial and emotional dysfunctions are mainly attributed to the decrease of apoptotic hippocampal neurons. However, to fully appreciate these results, the following points should be considered. First, AKT signaling itself is important for learning and memory $(51,52)$. In addition to reducing neuronal apoptosis, the AKT activity restored by dexmedetomidine exposure may participate in the improvements of cognitive deficits. Second, 5 or $10 \mu \mathrm{g} / \mathrm{kg}$ of dexmedetomidine was reported to improve spatial working memory in $\alpha_{2 C}$-adrenoceptor knockout mice (53). In addition, repeated administration of dexmedetomidine demonstrated decreased anxiety-like behaviors, impaired fear conditioning memory and improved spatial cognitive impairments in post-traumatic stress disorder model (54). Therefore, the amelioration of spatial and emotional disorders improved by dexmedetomidine administration may be multifactorial, and further studies are necessary to identify the relationship between consecutive dexmedetomidine exposure and long-term cognitive effects in the model of SAE.

Finally, one limitation of the present study was that the concentration of dexmedetomidine was not measured in the brain. A recent study determined the plasma and brain pharmacokinetics of dexmedetomidine in neonatal rats, and demonstrated the corresponding correlations between brain and plasma dexmedetomidine concentrations in the inset. Uptake of dexmedetomidine from the brain was rapid, and equilibration between plasma and brain was apparent by 15 min after dexmedetomidine injection (38). In summary, the present study suggested that application of dexmedetomidine could decrease neuronal apoptosis and increase neuronal survival in vitro and in vivo. It was also demonstrated that dexmedetomidine treatment markedly ameliorated the spatial and emotional dysfunctions induced by systemic sepsis. Taken together, the results suggested that application of dexmedetomidine could improve neuronal apoptosis and cognitive disorders in septic model.

\section{Acknowledgements}

The authors thank Professor Shoulin Zhang (Tianjin Medical University) for kind assistance in investigating behavior performance.

\section{Funding}

This project was supported by Tianjin Medical University (grant no. 2017-FM-00016).

\section{Availability of data and materials}

The datasets used and/or analyzed during the current study are available from the corresponding author on reasonable request.

\section{Authors' contributions}

LY designed the present study and prepared the draft manuscript. XC prepared the primary neuronal cultures and conducted cell transfection. HJ performed all the molecular biological experiments.SG performed the behavioral study, and data collection and analysis.

\section{Ethics approval and consent to participate}

All studies performed on animals were approved by the local Institutional Animal Care and Use Committee (Tianjin Baodi Hospital, Baodi Clinical College of Tianjin Medical University, Tianjin, China). All animal experiments complied with the ARRIVE guidelines and the National Institutes of Health guide for the care and use of Laboratory animals (NIH Publications no. 8023, revised 1978) (24).

\section{Patient consent for publication}

Not applicable.

\section{Competing interests}

The authors declare that they have no competing interests.

\section{References}

1. Vincent JL, Sakr Y, Sprung CL, Ranieri VM, Reinhart K, Gerlach H, Moreno R, Carlet J, Le Gall JR, Payen D, et al: Sepsis in European intensive care units: Results of the SOAP study. Crit Care Med 34: 344-353, 2006.

2. Iacobone E, Bailly-Salin J, Polito A, Friedman D, Stevens RD and Sharshar T: Sepsis-associated encephalopathy and its differential diagnosis. Crit Care Med 37 (10 Suppl): S331-S336, 2009.

3. Comim CM, Rezin GT, Scaini G, Di-Pietro PB, Cardoso MR, Petronilho FC, Ritter C, Streck EL, Quevedo J and Dal-Pizzol F: Mitochondrial respiratory chain and creatine kinase activities in rat brain after sepsis induced by cecal ligation and perforation. Mitochondrion 8: 313-318, 2008.

4. d'Avila JC, Santiago AP, Amâncio RT, Galina A, Oliveira MF and Bozza FA: Sepsis induces brain mitochondrial dysfunction. Crit Care Med 36: 1925-1932, 2008.

5. Weberpals M, Hermes M, Hermann S, Kummer MP, Terwel D, Semmler A, Berger M, Schäfers M and Heneka MT: NOS2 gene deficiency protects from sepsis-induced long-term cognitive deficits. J Neurosci 29: 14177-14184, 2009.

6. Hall JE, Uhrich TD, Barney JA, Arain SR and Ebert TJ: Sedative, amnestic, and analgesic properties of small-dose dexmedetomidine infusions. Anesth Analg 90: 699-705, 2000. 
7. Arcangeli A, D'alo C and Gaspari R: Dexmedetomidine use in general anaesthesia. Current Drug Targets 10: 687-695, 2009.

8. Pandharipande PP, Pun BT, Herr DL, Maze M, Girard TD, Miller RR, Shintani AK, Thompson JL, Jackson JC, Deppen SA, et al: Effect of sedation with dexmedetomidine vs lorazepam on acute brain dysfunction in mechanically ventilated patients: The MENDS randomized controlled trial. JAMA 298: 2644-2653, 2007.

9. Martin E, Ramsay GJ, Mantz J and Sum-Ping ST: The role of the alpha2-adrenoceptor agonist dexmedetomidine in postsurgical sedation in the intensive care unit. J Intensive Care Med 18 29-41, 2003.

10. Yang CL, Chen CH, Tsai PS, Wang TY and Huang CJ: Protective effects of dexmedetomidine-ketamine combination against ventilator-induced lung injury in endotoxemia rats. J Surg Res 167: e273-e281, 2011.

11. Okada H, Kurita T, Mochizuki T, Morita K and Sato S: The cardioprotective effect of dexmedetomidine on global ischaemia in isolated rat hearts. Resuscitation 74: 538-545, 2007.

12. Kocoglu H, Ozturk H, Ozturk H, Yilmaz F and Gulcu N: Effect of dexmedetomidine on ischemia-reperfusion injury in rat kidney: A histopathologic study. Ren Fail 31: 70-74, 2009.

13. Engelhard K, Werner C, Eberspacher E, Bachl M, Blobner M, Hildt E, Hutzler P and Kochs E: The effect of the alpha2-agonist dexmedetomidine and the $\mathrm{N}$-methyl-D-aspartate antagonist $\mathrm{S}(+)$-ketamine on the expression of apoptosis-regulating proteins after incomplete cerebral ischemia and reperfusion in rats. Anesth Analg 96: 524-531, 2003.

14. Hanci V, Erol B, Bektas S, Mungan G, Yurtlu S, Tokgöz H, Can M and Ozkoçak Turan I: Effect of dexmedetomidine on testicular torsion/detorsion damage in rats. Urol Int 84: 105-111, 2010.

15. Downward J: PI 3-kinase, AKT and cell survival. Semin Cell Dev Biol 15: 177-182, 2004.

16. Gass P, Schröder H, Prior P and Kiessling M: Constitutive expression of heat shock protein 90 (HSP90) in neurons of the rat brain. Neurosci Lett 182: 188-192, 1194.

17. Sato S, Fujita N and Tsuruo T: Modulation of AKT kinase activity by binding to Hsp90. Proc Natl Acad Sci USA 97: 10832-10837, 2000.

18. Li X, Luo R, Jiang R, Meng X, Wu X, Zhang S and Hua W: The role of the Hsp90/AKT pathway in myocardial calpain-induced caspase-3 activation and apoptosis during sepsis. BMC Cardiovas Dis 13: 8, 2013.

19. Shen E, Fan J and Peng T: Glycogen synthase kinase-3beta suppresses tumor necrosis factor-alpha expression in cardiomyocytes during lipopolysaccharide stimulation. J Cell Biochem 104 329-338, 2008.

20. Basso AD, Solit DB, Chiosis G, Giri B, Tsichlis P and Rosen N: AKT forms an intracellular complex with heat shock protein 90 (Hsp90) and Cdc37 and is destabilized by inhibitors of Hsp90 function. J Biol Chem 277: 39858-39866, 2002.

21. Zhang R, Luo D, Miao R, Bai L, Ge Q, Sessa WC and Min W: Hsp90-AKT phosphorylates ASK1 and inhibits ASK1-mediated apoptosis. Oncogene 24: 3954-3963, 2005.

22. Zhu YM, Wang CC, Chen L, Qian LB, Ma LL, Yu J, Zhu MH, Wen CY, Yu LN and Yan M: Both PI3K/AKT and ERK1/2 pathways participate in the protection by dexmedetomidine against transient focal cerebral ischemia/reperfusion injury in rats. Brain Res 1494: 1-8, 2013.

23. McGrath JC, Drummond GB, McLachlan EM, Kilkenny C and Wainwright CL: Guidelines for reporting experiments involving animals: The ARRIVE guidelines. Br J Pharmacol 160: 1573-1576, 2010

24. Bayne K: Revised guide for the care and use of laboratory animals available. American Physiological Society. Physiologist 39: 199, 208-211, 1996

25. Kaech S and Banker G: Culturing hippocampal neurons. Nat Protoc 1: 2406-2415, 2006.

26. Huang X, Venet F, Wang YL, Lepape A, Yuan Z, Chen Y, Swan R, Kherouf H, Monneret G, Chung CS and Ayala A: PD-1 expression by macrophages serves a pathologic role in altering microbial clearance and the innate inflammatory response to sepsis. Proc Natl Acad Sci USA 106: 6303-6308, 2009.

27. Ritter C, Andrades ME, Reinke A, Menna-Barreto S, Moreira JC and Dal-Pizzol F: Treatment with Nacetylcysteine plus deferoxamine protects rats against oxidative stress and improves survival in sepsis. Crit Care Med 32: 342-249, 2004.

28. Vorhees CV and Williams MT: Morris water maze: Procedures for assessing spatial and related forms of learning and memory. Nat Protoc 1: 848-858, 2006
29. Satomoto M, Satoh Y, Terui K, Miyao H, Takishima K, Ito M and Imaki J: Neonatal exposure to sevoflurane induces abnormal social behaviors and deficits in fear conditioning in mice. Anesthesiology 110: 628-637, 2009.

30. Brealey D, Karyampudi S, Jacques TS, Novelli M, Stidwill R, Taylor V, Smolenski RT and Singer M: Mitochondrial dysfunction in a long-term rodent model of sepsis and organ failure. Am J Physiol Regul Integr Comp Physiol 286: R491-R497, 2004.

31. Kamibayashi $\mathrm{T}$ and Maze M: Clinical uses of alpha2-adrenergic agonists. Anesthesiology 93: 1345-1349, 2000.

32. Siami S, Annane D and Sharshar T: The encephalopathy in sepsis. Crit Care Clin 24: 67-82, 2008

33. Pytel P and Alexander JJ: Pathogenesis of septic encephalopathy. Cur Opin Neurol 22: 283-287, 2009.

34. Semmler A, Hermann S, Mormann F, Weberpals M, Paxian SA, Okulla T, Schäfers M, Kummer MP, Klockgether T and Heneka MT: Sepsis causes neuroinflammation and concomitant decrease of cerebral metabolism. J Neuroinflam 5: 38, 2008.

35. Taccone FS, Su F, Pierrakos C, He X, James S, Dewitte O, Vincent JL and De Backer D: Cerebral microcirculation is impaired during sepsis: An experimental study. Crit Care 14: R140, 2010.

36. Schoeler M, Loetscher PD, Rossaint R, Fahlenkamp AV, Eberhardt G, Rex S, Weis J and Coburn M: Dexmedetomidine is neuroprotective in an in vitro model for traumatic brain injury. BMC Neurol 12: 20, 2012.

37. Degos V, Charpentier TL, Chhor V, Brissaud O, Lebon S, Schwendimann L, Bednareck N, Passemard S, Mantz J and Gressens P: Neuroprotective effects of dexmedetomidine against glutamate agonist-induced neuronal cell death are related to increased astrocyte brain-derived neurotrophic factor expression. Anesthesiology 118: 1123-1132, 2013.

38. McAdams RM, McPherson RJ, Kapur R, Phillips B, Shen DD and Juul SE: Dexmedetomidine reduces cranial temperature in hypothermic neonatal rats. Pediatr Res 77: 772-778, 2015.

39. Park JH, Derry K and Owens R: 896: Dexmedetomidine as adjunctive sedation in mechanically ventilated patients. Crit Care Med 47: 427, 2019.

40. Martini M, De Santis MC, Braccini L, Gulluni F and Hirsch E: PI3K/AKT signaling pathway and cancer: An updated review. Ann Med 46: 372-383, 2014.

41. Dudek H, Datta SR, Franke TF, Birnbaum MJ, Yao R, Cooper GM, Segal RA, Kaplan DR and Greenberg ME: Regulation of neuronal survival by the serine-threonine protein kinase AKT. Science 275: 661-665, 1997.

42. Li Y,Zeng M, Chen W, Liu C, Wang F, Han X, Zuo Z and Peng S: Dexmedetomidine reduces isofluraneinduced neuroapoptosis partly by preserving PI3K/AKT pathway in the hippocampus of neonatal rats. PLoS One 9: e93639, 2014.

43. Farghaly HS, Mahmoud AM and Abdel-Sater KA: Effect of dexmedetomidine and cold stress in a rat model of neuropathic pain: Role of interleukin- 6 and tumor necrosis factor- $\alpha$. Eur J Pharmacol 776: 139-145, 2016.

44. Li J, Chen Q, He X, Alam A, Ning J, Yi B, Lu K and Gu J: Dexmedetomidine attenuates lung apoptosis induced by renal ischemia-reperfusion injury through $\alpha_{2} \mathrm{AR} / \mathrm{PI} 3 \mathrm{~K} / \mathrm{AKT}$ pathway. J Transl Med 16: 78, 2018

45. Ibacache M, Sanchez G, Pedrozo Z, Galvez F, Humeres C, Echevarria G, Duaso J, Hassi M, Garcia L, Díaz-Araya G and Lavandero S: Dexmedetomidine preconditioning activates pro-survival kinases and attenuates regional ischemia/reperfusion injury in rat heart. Biochim Biophys Acta 1822: 537-545, 2012.

46. Karkoulias G, Mastrogianni O, Lymperopoulos A, Paris H and Flordellis C: alpha(2)-Adrenergic receptors activate MAPK and AKT through a pathway involving arachidonic acid metabolism by cytochrome P450-dependent epoxygenase, matrix metalloproteinase activation and subtype-specific transactivation of EGFR. Cell Signal 18: 729-739, 2006

47. Cai W, Rudolph JL, Harrison SM, Jin L, Frantz AL, Harrison DA and Andres DA: An evolutionarily conserved Rit GTPase-p38 MAPK signaling pathway mediates oxidative stress resistance. Mol Biol Cell 22: 3231-3241, 2011.

48. Koh PO: Nicotinamide attenuates the ischemic brain injury-induced decrease of AKT activation and Bad phosphorylation. Neurosci Lett 498: 105-109, 2011.

49. Mendoza MC, ErEE and Blenis J: The Ras-ERK and PI3K-mTOR pathways: Cross-talk and compensation. Trends Biochem Sci 36: 320-328, 2011. 
50. Parcellier A, Tintignac LA, Zhuravleva E and Hemmings BA: PKB and the mitochondria: AKTing on apoptosis. Cell Signal 20: 21-30, 2008

51. Franke TF: PI3K/Akt: Getting it right matters. Oncogene 27: 6473-6488, 2008.

52. Horwood JM, Dufour F, Laroche S and Davis S: Signalling mechanisms mediated by the phosphoinositide 3-kinase/AKT cascade in synaptic plasticity and memory in the rat. Eur J Neurosci 23: 3375-3384, 2006.
53. Björklund M, Siverina I, Heikkinen T, Tanila H, Sallinen J, Scheinin $M$ and Riekkinen P Jr: Spatial working memory improvement by an alpha2-adrenoceptor agonist dexmedetomidine is not mediated through alpha2C-adrenoceptor. Prog Neuropsychopharmacol Biol Psychiatry 25: 1539-1554, 2001.

54. Ji MH, Jia M,Zhang MQ, Liu WX, Xie ZC, Wang ZY and Yang JJ: Dexmedetomidine alleviates anxiety-like behaviors and cognitive impairments in a rat model of post-traumatic stress disorder. Prog Neuropsychopharmacol Biol Psychiatry 54: 284-288, 2014. 